\title{
USO DEL TIEMPO LIBRE DE LAS PERSONAS ADULTAS MAYORES RESIDENTES EN ALBERGUES
}

\section{USE OF FREE TIME OF THE OLDER ADULT RESIDENTS IN SHELTERS}

\author{
Derby Muñoz Rojas* \\ Jennifer Hughes Cartigny**
}

RESUMEN

El estudio tuvo como objetivo establecer la situación actual de la utilización del tiempo libre de las personas adultas mayores residentes en albergues ${ }^{1}$. Fue de tipo cualitativo con un enfoque fenomenológico, con una muestra de siete albergues. Para recolectar la información se utilizaron entrevistas y observaciones. Se encontró que no existe un uso creativo del tiempo libre por parte de los y las residentes, hay una diferencia entre las políticas sociales establecidas para la recreación en los albergues y la realidad que se vive en los mismos.

PALABRAS CLAVE: VEJEZ * ALBERGUES * TIEMPO LIBRE * RECREACIÓN

\section{ABSTRACT}

The study had as aim to establish the current situation of the utilization of the free time of the older adult residents in shelters. It was qualitative with a phenomenological approach, with a sample of seven shelters. For collect information, we used interviews and observations, in each center. It was found that there is not a creative use of leisure time by older people living in shelters, there is a difference between social policies established for recreation in the shelters and the reality that they live in the shelters.

KEYWORDS: OLD AGE * SHELTERS * LEISURE * RECREATION

Escuela de Enfermería, Universidad de Costa Rica. derby.munoz@ucr.ac.cr

** Escuela de Enfermería, Universidad de Costa Rica jenniferhughes9@hotmail.com

1 El término albergue corresponde a una modalidad específica de los centros de atención para persona adulta mayor (Ley 7935). Sin embargo, en algunos contextos se utiliza el término albergue y hogar como sinónimos; para efectos propios del estudio se conceptualizó al albergue en función de modalidad por sí mismo y no como referente para el término hogar. 


\section{INTRODUCCIÓN}

La disminución en las tasas de natalidad, el mejoramiento en la expectativa de vida de las personas y el envejecimiento acelerado en la población, han contribuido enormemente a generar cambios sociodemográficos a nivel de todo el mundo. Dentro de estos se encuentra el incremento en la cantidad de personas adultas mayores, caracterizándose no solo por el aumento en la cantidad de años vividos, sino también en la cantidad de personas que logran alcanzar la longevidad.

Estas modificaciones sociodemográficas vienen acompañadas de una serie de cambios a nivel social, económico y cultural, para lo cual los individuos y la sociedad como tal han tenido que generar estrategias con el fin de controlar el impacto de estos cambios. Dentro de estas estrategias se encuentra la creación de instituciones de bienestar social, como los albergues, en los cuales se busca mantener una adecuada calidad de vida al satisfacer las necesidades básicas de algunos de los individuos de este colectivo.

El envejecimiento como proceso inherente a la vida del ser humano, debe ser visto desde un enfoque integral. Rodríguez et ál. (2000) y Millán (2006), exponen que el envejecimiento comprende modificaciones morfológicas, fisiológicas, bioquímicas, psicológicas y sociales según el contexto temporo-espacial en el que se desarrolló el individuo en las etapas anteriores a la adultez mayor y que aparecen como consecuencia del paso del tiempo. Dichos cambios se insinúan o aceleran después de haber alcanzado el crecimiento y madurez, alrededor de los 30 años de edad; a partir de entonces como regla general, cada año se pierde el $1 \%$ de las potencialidades y reservas fisiológicas del organismo promedio.

Desde este enfoque integral, Burke y Walsh (1998) plantean que existen teorías que explican estos cambios; por ejemplo, la teoría del desligamiento, la de la actividad y la del envejecimiento programado. La mayoría de las teorías comparten que las personas sufren modificaciones en sus dimensiones, dentro de estas, Millán (2006) plantea que a nivel psicológico el ser humano puede experimentar cambios en su cognición y en su autopercepción; Hidalgo (2001) expone a nivel social que la persona sufre modificaciones en su rol social; mientras que Wilmore y Costill (2004), mencionan que a nivel físico la persona experimenta cambios al envejecer, dentro de estos se encuentra la pérdida en la funcionalidad neural, cambios vasculares y respiratorios.

Paralelo al proceso de envejecimiento e inherente a la condición de todo ser humano, toda persona sigue manteniendo una serie de necesidades básicas para poder seguir subsistiendo. Buendía (1996) plantea que para satisfacer las necesidades básicas de la población adulta mayor, hay ocasiones en que deben de ser institucionalizados(as) como en hospitales, residencias, hogares y albergues, en los cuales a través de un proceso de acompañamiento, se busca cumplir con todas las necesidades básicas, tales como alimentación, hospedaje, vestido y recreación.

Goffman citado por Hidalgo (2001), define a la institucionalización como un proceso social de ubicar a ciertas personas bajo la jurisdicción de una institución formal o semiformal, cerrada o semicerrada, en donde esta ubicación conlleva la búsqueda del bienestar de la persona a partir de la satisfacción de las necesidades básicas de alimento, recreación, vestido, vivien$\mathrm{da}$, control de salud y cualquier actividad que permita su potencialización como persona.

En las Normas para la Acreditación de Establecimientos de Atención Integral a la Persona Adulta Mayor (Centro Diurno- Hogar), el Ministerio de Salud (2006) define los albergues como establecimientos donde se brinda atención integral a las y los adultos mayores de forma permanente, a partir de tres personas, por lo que requiere de personal de atención directa las 24 horas para poder realizar las actividades de la vida diaria.

En el informe "Contexto y Perfil de las Instituciones de Bienestar Social en Costa Rica" se define al albergue como una variante de la modalidad de hogar para adultas y adultos mayores, más específicamente como "establecimientos de carácter privado, sin fines de lucro, en donde viven personas adultos/as mayores en forma permanente $y$ en los cuales se brindan 
servicios integrales, como respuesta a problemas de abandono familiar, social y económico" (Valverde, 2004: 13).

El Ministerio de Salud (2004) menciona que en esta modalidad de centros, la persona adulta mayor es autónoma, se vale por sí misma, tiene áreas comunes con otras y otros adultos mayores, pero a la vez tiene privacidad. Los servicios básicos que brinda un albergue son: alimentación, lavandería, habitación, actividades recreativas, ocupacionales y culturales, actividades físicas, estimulación mental y contacto social; e incluso, designa a la comunidad como partícipe del funcionamiento del centro.

Estas instituciones son en muchas ocasiones de bienestar social, sin embargo, su funcionamiento, según Millán (2006) se encuentra regulado por leyes, reglamentos y normas de acuerdo con cada país, que incluyen desde las acciones que se realizan con los adultos mayores hasta el perfil del personal que labora y los espacios y equipos con que debe trabajar.

Todas las normativas, de acuerdo con la Ley Integral para la Persona Adulta Mayor (Ley 7935) de la Asamblea Legislativa (1999) van orientadas a satisfacer las necesidades básicas, a través de las cuales las personas adultas mayores logran experimentar una adecuada calidad de vida a nivel psicológico, físico y social, la que es fundamental para lograr un envejecimiento exitoso. Este envejecimiento exitoso, según Brigeiro (2005) debe ser el proceso que experimente la misma persona con el fin de envejecer de la manera más satisfactoria posible.

Fernández (1999) aporta a la definición anterior que el envejecimiento exitoso es un proceso de optimización de oportunidades para luchar por la salud, por la participación activa en la vida y por la seguridad de alcanzar calidad de vida cuando el ser humano envejece, que incluye tres componentes principales: disminuir el riesgo de padecer enfermedades o invalidez, tener un tono físico adecuado funcional y cognitivo, tener una participación activa en la sociedad. La convergencia de estas temáticas ha sido de interés para diferentes grupos que han elaborado investigaciones en torno a esta.

A nivel internacional y nacional, se han registrado múltiples estudios, informes $y$ documentos relacionados con la población de personas adultas mayores, sin embargo, muy pocos se han centrado en el contexto específico de las instituciones de bienestar social y específicamente en los albergues.

En Costa Rica, Valverde (2004) elaboró para el Consejo Nacional de la Persona Adulta Mayor (CONAPAM) el informe "Contexto y perfil de las instituciones de bienestar social (IBS) para la atención de las personas adultas mayores", en donde define que para el 2004 habían diez albergues habilitados y en funcionamiento; el autor (2004) plantea dos situaciones importantes; en primer lugar, para efectos del informe, los albergues en sus diferentes modalidades no fueron incluidos dentro de los datos obtenidos; en segundo lugar, en cuanto a la recreación dice que el $72 \%$ de las instituciones de bienestar social en la modalidad de hogar, tienen acceso a actividades de este tipo, pero no especificó cuáles tipos de actividades, frecuencia y persona encargada, entre otras características. También señala que existen organizaciones no gubernamentales como la Asociación Gerontológica Costarricense (AGECO) que poseen programas específicos según la necesidad comunal.

Para Costa Rica, el Proyecto Estado de la Nación (2007), plantea que en los últimos años se ha presentado una mejoría en la cobertura de los servicios de salud para las personas adultas mayores, pero no se enfatiza en la satisfacción de la necesidad de la recreación.

Sin embargo, en las diferentes modalidades de las instituciones en las que residen las personas adultas mayores, existe el planteamiento de que se deben realizar distintas actividades a través de las que logren satisfacer sus necesidades básicas. Durante el día también existen espacios de tiempo libre en los cuales las personas adultas mayores pueden realizar diferentes actividades para mejorar su calidad de vida, entre las que sobresale el ejercicio y la recreación, en donde la recreación es entendida como:

Un proceso de acción participativa $y$ dinámica que facilita entender la vida como una vivencia de disfrute, creación 
y libertad, en el pleno desarrollo de las potencialidades del ser humano para su realización y mejoramiento de la calidad de vida individual y social, mediante la práctica de actividades físicas, intelectuales o de esparcimiento (Rico, 1999: 14).

En Costa Rica, la Organización Panamericana de la Salud (OPS) (2004), en su estudio determinó que existen diferentes instituciones que poseen programas orientados hacia el uso creativo del tiempo libre, por medio de diferentes estrategias como la recreación y el ejercicio físico; por ejemplo, la Caja Costarricense del Seguro Social (CCSS) tiene el "Programa Ciudadano de Oro", que incluye cursos, talleres $y$ charlas, preparación para la jubilación y convenios preferenciales que permiten descuentos en transporte público, adquisición de artículos y entradas a eventos, entre otros.

En Costa Rica, pese a que se han seguido varias líneas de investigación relacionada con el movimiento humano en personas adultas mayores institucionalizadas, en la modalidad de albergues no hay evidencias encontradas o datos de esta población, especialmente en estilos de vida, hábitos de recreación y tiempo libre; por esta razón fue necesario realizar un diagnóstico nacional de albergues sobre la manera en que las personas adultas mayores residentes utilizan su tiempo libre.

\section{METODOLOGÍA}

Esta investigación se desarrolló utilizando un enfoque cualitativo, de tipo fenomenológico, debido a que este permitió captar las realidades cuya naturaleza y estructura particular obedecieron a un marco de referencia interno del sujeto que las vivió y experimentó. El espacio temporal fue de tipo transversal, durante el periodo de tiempo comprendido entre septiembre y diciembre del año 2008.

LUGARES: al ser un estudio nacional se consideraron los diez albergues colectivos para personas adultas mayores en Costa Rica, habilitados por el Ministerio de Salud Pública y el Instituto Mixto de Ayuda Social; de los cuales por selección dirigida (no probabilística) se seleccionaron siete instituciones, uno de Limón, uno de Guanacaste, uno de Puntarenas, uno de Alajuela y tres de San José. Considerando para el muestreo a) la dinámica propia de cada albergue; b) la autorización respectiva para realizar el estudio y c) el criterio de saturación de información.

PROTAGONISTAS: la población total fue de 14 personas, divididas en dos estratos; en primer lugar, el de personas adultas mayores residentes compuesto por tres sujetos $(n=3)$ de sexo femenino y cuatro $(n=4)$ de sexo masculino, con una edad promedio para toda la muestra de 75,0 \pm 7,6 años; el segundo estrato de trabajadores de la institución, compuesto por siete sujetos $(n=7)$, de sexo femenino con una edad promedio de 39,1 \pm 10,5 años.

Se decidió utilizar una muestra para la investigación por tres razones: 1) debido al componente geográfico; 2) debido a que la cantidad de personas que componen la población total es muy elevada $y$ de muy diferentes características, lo que pudo dificultar la obtención completa de la información; 3) por la restricción o no anuencia a participación en el estudio por parte de algunos albergues. Cada sujeto fue informado acerca de los alcances y limitaciones del estudio, así como, de los posibles beneficios directos e indirectos $y$ situaciones no beneficiosas relacionadas con su participación en la investigación, posteriormente se realizó la aplicación del consentimiento informado.

INSTRUMENTOS Y MATERIALES: la recolección de la información se llevó a cabo por medio de tres instrumentos, validado previamente por dos técnicas diferentes, en primer lugar, por el juicio de expertos, quienes revisaron y corrigieron los documentos y luego, por la aplicación de cada instrumento en una población con características similares. Los instrumentos fueron diseñados a partir de los documentos del Ministerio de Salud Pública; "Normas para la Acreditación de Establecimientos de Atención Integral a la Persona Adulta Mayor (Centro Diurno-Hogar) del Ministerio de Salud de Costa Rica (2006)" y el "Cuestionario de Evaluación para la Acreditación de Establecimientos de Atención Integral a la Persona Adulta Mayor Centro Diurno y Hogar del Ministerio de Salud de Costa Rica (2006)". Tomando en cuenta los 
apartados específicos con la temática de la recreación.

$\diamond \quad$ Observación no participante: se estructuró en cinco categorías (participantes residentes, participantes funcionarios, espacios físicos e infraestructura, materiales $y$ equipos, actividades recreativas) con un total de 50 aspectos a observar por medio de una escala dicotómica de presente $y$ ausente, con un espacio para comentarios.

$\diamond \quad$ Entrevista semi estructurada para el personal: se organizó en preguntas abiertas y cerradas por medio de nueve categorías (datos socio demo-gráficos, datos de relación con el centro, información acerca del centro, distribución del tiempo en actividades, opciones de actividades para utilizar el tiempo libre, espacios para la recreación, equipos y materiales, personas que implementan las actividades $y$ proyectos futuros) con un total de 25 preguntas.

$\diamond \quad$ Entrevista semi estructurada para los $y$ las residentes: se organizó en pre-guntas abiertas y cerradas por medio de nueve categorías (datos socio demográficos, datos de relación con el centro, información acerca del centro, distribución del tiempo en actividades, opciones de actividades para utilizar el tiempo libre, espacios para la recreación, equipos y materiales, personas que implementan las actividades $y$ proyectos futuros) con un total de 24 preguntas.

PROCEDIMIENTO: inicialmente se ubicó y contactó a cada una de las personas encargadas de la administración de cada albergue, posteriormente se seleccionó los lugares para hacer las visitas. Luego de la calendarización de las visitas se viajó a cada institución y con las personas seleccionadas con la ayuda de la administración y el personal de enfermería de cada centro, se realizó la aplicación de las dos entrevistas; una vez finalizadas estas, se procedió a realizar la observación no participante por un espacio de dos a cuatro horas en cada lugar, considerando todos los espacios permitidos de los albergues.

La información recolectada se estratificó de acuerdo con las categorías de características socio demográficas, políticas institucionales acerca del funcionamiento de cada institución, distribución o uso del tiempo durante el día por parte de los y las residentes, uso creativo del tiempo libre, actividades recreativas, información acerca de las personas que brindan y participan en actividades de recreación durante el tiempo libre, espacios para la recreación $y$ datos acerca de posibles actividades futuras para recreación durante el tiempo libre. Posteriormente, se realizó la triangulación de los datos en cada categoría y finalmente se analizó la información.

\section{RESULTADOS}

En forma organizada de acuerdo con las categorías del estudio se presentan a continuación los resultados obtenidos.

Socio demográficamente se encontró que todos los sujetos del estrato de funcionarias residían en las cercanías de los respectivos albergues en los cuales laboran. Con respecto a la información laboral, en promedio las funcionarias poseen $4,9 \pm 4.0$ años de laborar para la institución; señalan como funciones por la que fueron contratadas: aseo e higiene de los residentes, limpieza y orden de la institución, preparación y cocción de los alimentos, servicios de enfermería y administración; sin embargo, todas coinciden en que son polifuncionales debido a que deben ejecutar muchas $y$ diferentes tareas durante el día.

Además, poseen una formación académica variada, la cual se caracterizó porque tres de las participantes no poseen educación formal completa de ningún tipo, dos poseen primaria, una finalizó la secundaria y solamente una posee un nivel universitario, de bachillerato en enfermería. De estas, cinco poseen educación no formal en diferentes aspectos como: manipulación de alimentos, salud ocupacional, primeros auxilios y contabilidad; todas han recibido alguna capacitación por parte del albergue en temas relacionados con el cuidado de las personas adultas mayores; cinco de los 
sujetos han recibido alguna capacitación acerca del cuidado de las y los residentes por parte del Hospital Nacional de Geriatría y Gerontología Dr. Raúl Blanco Cervantes.

En el estrato de personas adultas mayores residentes, todos los sujetos manifestaron que su lugar de procedencia antes de ingresar a residir en la institución era en las cercanías de la comunidad donde se encuentra el centro y en promedio han residido en la institución 5,3 \pm 4,0 años. Además, seis de los sujetos no tienen primaria completa y solo una residente finalizó dicho nivel.

\section{A.1. MISIÓN, VISIÓN Y OBJETIVOS DE FUNCIONAMIENTO}

Con respecto a esta categoría existe un consenso general en ambos estratos al verbalizar que la misión, la visión y el propósito por los cuales funcionan los albergues para personas adultas mayores es el de satisfacer las necesidades básicas. Sin embargo, dentro de lo expresado por todos los sujetos, no se incluye a la recreación como una de estas necesidades. A continuación se enlistan algunos de los comentarios realizados por los sujetos al respecto (Fuente: entrevistas semi estructuradas para funcionarias realizadas en diciembre de 2008; material original disponible. Fuente: entrevistas semi estructuradas para residentes realizadas en diciembre de 2008; material original disponible):

-... una casita para que no les falte comidita, ropita, medicinas y todo lo que necesiten, porque no tienen donde pasar lo que Diosito quiera (Sujeto 1, albergue 1 , estrato funcionarios).

-... ser un servicio a la comunidad... donde nunca les va a faltar su cafecito, pancito, sus frijolitos... se les da todo lo que les haga falta (Sujeto 2, albergue 2, estrato funcionarios).

- Ayudar a los viejitos que les falta un lugar para dormir, o que no tienen familia o nadie que les lave ni les planche o les cocine (Sujeto 4, albergue 4, estrato funcionarios).

-... centro, asociación organizada para satisfacer las necesidades de los ancianos del cantón... como tiempos de comida, aseo e higiene, medicamentos, $y$ control médico (Sujeto 5, albergue 5, estrato funcionarios).

-... darnos un lugar para vivir lo que nos queda, con comida, ropa nueva, medicinas, doctora... (Sujeto 1, albergue 1 , estrato residentes).

-Un hospital para la gente enferma... dan comida, doctor, nos bañan, nos llevan a la iglesia, a la clínica... (Sujeto 4, albergue 4, estrato residentes).

-... un cuartito para pasar tranquilo... regalarnos la comida, la dormida, hasta la camita (Sujeto 5, albergue 5, estrato residentes).

\section{A.2. CANTIDAD DE TIEMPO LIBRE}

De acuerdo con la información suministrada por los y las participantes del estrato de funcionarias y del estrato de residentes, las personas adultas mayores que residen en los albergues, distribuyen el día de la siguiente manera, utilizan de 8 a 12 horas para dormir, en las cuales se incluyen periodos del día como la siesta después del almuerzo.

Las funcionarias y las personas adultas mayores expresaron que para bañarse $y$ vestirse, los y las residentes necesitan un tiempo de 25 a 40 minutos, considerando el nivel de funcionalidad de cada persona (Fuente: entrevista semi estructurada para funcionarios realizada al sujeto 3 del albergue 3, diciembre de 2008, material original disponible).

Considerando que en todos los albergues se tiene una distribución de cinco a seis tiempos de alimentación durante un día, se encontró que el tiempo total de alimentación durante un día es de una hora a una hora y media, 
distribuidos en " 15 minutos para desayunar, 10 minutos en la merienda, 15 a 25 almorzando, 10 a 15 minutos tomando café con un pedazo de pan, y 20 minutos tomándose el atol y la cena" (Fuente: entrevista semi estructurada para residentes realizada al sujeto 7 del albergue 7 , diciembre de 2008, material original disponible).

Con respecto al tiempo empleado por los y las residentes para realizar actividades propias del centro como ir a misa, reuniones, tareas asignadas como barrer y lavar platos, entre otros, se encontraron dos situaciones. Primero, en los centros ninguna persona residente posee tareas asignadas o funciones dentro de los albergues; si algún o alguna residente realiza alguna labor dentro del centro es porque ellos o ellas lo desean. Segundo, el tiempo para actividades propias de cada institución es muy variado, ya que de acuerdo con los datos recopilados, estas actividades varían de acuerdo al día de la semana; una funcionaria comenta que los horarios de los servicios religiosos varían por día de la semana; por ejemplo, los jueves se realiza la Hora Santa y los viernes una misa, el resto de días no hay actividades de este tipo, por lo que el total de horas del día destinado para estas diferentes actividades se encuentra entre una a dos horas (Fuente: entrevista semi estructurada para funcionarios realizada al sujeto 6 del albergue 6, 2008, material original disponible).

En las horas reportadas para la realización del ejercicio físico, la mayoría de sujetos coincidieron en que utilizan una hora diaria, aunque comentan que este espacio de tiempo se encuentra relacionado con la atención por parte del profesional de terapia física, ya que según lo manifestado, en esta sesión es el único momento en que se realiza ejercicio (Fuente: entrevista semi estructurada para funcionarios realizada al sujeto 1 del albergue 1, 2008, material original disponible).

De las horas del día destinadas para la recreación, se encontraron dos situaciones diferentes. Primero, la mayoría de los sujetos reportaron el mismo comportamiento con respecto a las horas que se utilizan para realizar ejercicio físico; una residente comenta que realizan jue- gos de mesa, dibujos y hacen casitas solo los martes, jueves y sábados con la terapeuta física (Fuente: entrevista semi estructurada para residentes realizada al sujeto 7 del albergue 7 , 2008, material original disponible). Segundo, existen dos casos con un comportamiento distinto; en el primero de ellos, los y las residentes asisten de lunes a viernes a un centro diurno de la comunidad, razón por la cual realizan actividades de ejercicio y recreación de 3 a 5 horas por día; en el otro de los casos, el albergue es un campo clínico para diferentes carreras de una universidad privada; dentro de las actividades que realizan las y los estudiantes hay de tipo recreativas y de ejercicio físico, entre otras; sin embargo, estas no tienen un horario establecido por lo que no es posible cuantificarlo o determinarlo con precisión.

En lo que concierne al tiempo libre, por medio de los datos obtenidos se cuantificó entre cinco a siete horas por día, $y$ depende directamente del día y del centro en particular. Todos los sujetos comentaron que este periodo de tiempo es principalmente en la tarde; una funcionaria plantea que los y las residentes no hacen nada después de la merienda y hasta el almuerzo, después del café y hasta el colado (Fuente: entrevista semi estructurada para funcionarios realizada al sujeto 5 del albergue 5 , 2008, material original disponible).

\section{A.3. ACTIVIDADES, PROGRAMAS Y PROYECTOS PARA EL USO CREATIVO DEL TIEMPO LIBRE}

Con respecto a la manera en como utilizan los y las residentes su tiempo libre durante el día, se encontró que existe el consenso entre los sujetos al plantear que la mayoría de residentes se dedican a estar sentados en hileras contra la pared, en un solo lugar, ya sea una silla, un sillón, una silla de ruedas, una banca o una mecedora, en donde pasan hablando entre ellos y ellas; algunos y algunas escuchan radio en especial emisoras musicales; otros $y$ otras se dedican a ver televisión. Una funcionaria comenta que las y los residentes se sientan toda la tarde a ver en la televisión programas variados como telenovelas, noticias, películas 
mexicanas, fábulas, partidos de fútbol, pero acota que en ocasiones desconoce si realmente se encuentran observando el programa de televisión o solamente están viendo la pantalla de la televisión sin poner atención (Fuente: entrevista semi estructurada para funcionarios realizada al sujeto 4 del albergue 4, 2008, material original disponible y observaciones no participante, 2008, material original disponible).

Todas las siete funcionarias concordaron al asegurar que durante el tiempo libre la gran mayoría de los y las residentes no hacen ningún tipo de actividad recreativa, o de ejercicio físico, por el contrario pasan ese tiempo sentados sin realizar nada; una funcionaria menciona que nunca hacen nada, incluso la mayoría pasa más dormida, aunque se encuentren sentados (Fuente: entrevista semi estructurada para funcionarios, 2008, material original disponible).

De los datos recolectados, se desprende que ese comportamiento se generaliza para todos los albergues y sujetos, con la excepción de entre uno y dos casos individuales por centro, en donde son una o dos personas que por iniciativa propia realizan alguna actividad recreativa; de las entrevistas se extraen los dos siguientes comentarios que ejemplifican este comportamiento (Fuente: entrevista semi estructurada para residentes, 2008, material original disponible):

Sujeto 1, albergue 1: -... Doña Ángeles pasa todo el día ocupada... lee La Nación, pasa pintando, ayuda a repartir colados... baila, camina, nunca está como los otros sentadotes roncando. En las tardes me pongo a coser, o a hacer manualidades...

Sujeto 6, albergue 6:-Salgo a dar una vuelta para no aburrirme, voy a la panadería... me pongo a regar los jardines, a botar maleza... es que me gusta mucho la jardinería y todo eso.

En todos los albergues la mayoría de las funcionarias $y$ de los $y$ las residentes, excepto una de las funcionarias, desconocen la existencia de posibles actividades futuras orientadas a la recreación de las personas; la única funcionaria que conocía la información comentó que existe un proyecto con una universidad para hacer del albergue un campo clínico, en el cual los y las estudiantes van a realizar diferentes actividades, entre estas, charlas, masajes, juegos y bailes, entre otras (Fuente: entrevista semi estructurada para funcionarios, 2008, material original disponible).

De la información obtenida se desprenden dos situaciones importantes, en el primer caso, durante el año las actividades recreativas tienen una estrecha relación con épocas festivas, o con trabajos o asignaciones académicas de diferentes instituciones, todos los sujetos comentaron que para la celebración del día del padre, de la madre, del niño y la niña, de la persona adulta mayor, navidad, siempre aparecen grupos que pasan unas horas con los $y$ las residentes, periodo durante el cual les entregan regalos, realizan bailes, cantan, presentan obras de teatro, entre otras actividades. Un residente comenta que para el día del padre siempre le regalan medias $y$ vienen a bailar con él (Fuente: entrevista semi estructurada para residentes, 2008, material original disponible). Una funcionaria menciona que debe hacer horarios para que varios grupos puedan venir en la semana de navidad porque todos quieren venir el sábado y el domingo antes de navidad $y$ es que quedan muy cansados porque bailan, juegan y comen mucho (Fuente: entrevista semi estructurada para funcionarios, 2008, material original disponible).

En segundo lugar, los sujetos expresaron que las actividades propias en las diferentes instituciones no son frecuentes, que por el contrario, en la mayoría de los días no hay ningún tipo de actividades que realizar. Ante esto un residente comenta que en el albergue no hay cosas para hacer fuera de ver televisión, leer el periódico y descansar en los sillones (Fuente: entrevista semi estructurada para residentes, 2008, material original disponible), mientras que una de las funcionarias mencionó que las actividades recreativas que ofrece la institución son esporádicas, pero que sí hay programadas durante el año, como los cumpleaños por décadas (se celebran los cumpleaños de los residentes que llegan a los 70, 80, 90, 100 años de 
edad) y los paseos anuales de verano (Fuente: entrevista semi estructurada para funcionarios, 2008, material original disponible).

Los sujetos, al momento del diagnóstico, al verbalizar las justificaciones por las cuales no se realizan actividades recreativas con más frecuencia, las orientaron en razones de tipo administrativo en primer lugar $y$ en segundo lugar, por falta de participación por parte de los y las residentes. Con respecto al manejo administrativo de las actividades de recreación, externaron como razones principales la falta de personal, los horarios de trabajo y la cantidad de labores que hay que realizar (Fuente: entrevista semi estructurada para funcionarios, 2008, material original disponible).

La falta de participación por parte de los y las residentes es justificada por los sujetos debido a la falta de interés por este tipo de actividades, por los sentimientos de cansancio $y$ aburrimiento, $y$ por la percepción inadecuada del tipo de actividades que se realizan durante el tiempo libre: -... estas mujeres nunca hacen nada $y$ si se ponen es a hablar mientras limpian, y a mí no me gusta hablar... el muchacho de terapia solo a pegar papel y a jugar bingo nos pone y eso no me gusta (Fuente: entrevista semi estructurada para residentes, 2008, material original disponible).

\section{A.4. PERSONAL ENCARGADO DE LA REALIZACIÓN DE LAS ACTIVIDADES RECREATIVAS}

En la mayoría de los albergues, los sujetos expresaron no conocer quién es la persona responsable de esas actividades, por ejemplo, un residente comenta que no sabe a cuál de las funcionarias se le paga por realizar actividades recreativas (Fuente: entrevista semi estructurada para residentes, 2008, material original disponible), una funcionaria mencionó que nadie se encarga de eso y que cuando alguien lo hace es por iniciativa propia, o porque llega a la institución a realizar alguna tarea como servicio comunal, o vienen de la iglesia (Fuente: entrevista semi estructurada para funcionarios, 2008, material original disponible).

Todos los sujetos comentaron que el o la profesional de terapia física realiza en algunas ocasiones actividades lúdicas o de recreación, pero que desconocen con qué fin utilizan las actividades de esta índole. Una de las funcionarias comentó que la terapeuta no tiene planes de trabajo, solo horarios de dos o tres días por semana, una hora a dos horas por cada persona, aunque también trabaja con varios al mismo tiempo (Fuente: entrevista semi estructurada para funcionarios, 2008, material original disponible).

En uno de los albergues, una universidad de modalidad privada se encuentra realizando algunas prácticas con algunos estudiantes, razón por la cual en este apartado un residente plantea que son las personas que vienen de afuera las que se encargan de realizar las actividades recreativas, como bailar, jugar bingo o bien, jugar con las cartas (Fuente: entrevista semi estructurada para residentes, 2008, material original disponible).

\section{A.5. RECURSOS MATERIALES Y CAPACIDAD ESTRUCTURAL Y FÍSICA PARA LA RECREACIÓN}

En todos los albergues visitados se encontró que poseen extensas áreas al aire libre, las que se caracterizan por poseer combinación entre áreas verdes y áreas pavimentadas. Un gran porcentaje de las áreas verdes se encuentran destinadas como jardines; estas áreas verdes poseen árboles grandes, pasillos bajo techo y lámparas, entre otros dispositivos. En uno solo de los albergues existe un espacio en el exterior que funciona como gallinero, con un corral para un cerdo y un espacio para un huerto; en el resto de instituciones no se observó este tipo de espacios.

Los espacios al aire que están pavimentados se caracterizan en su mayoría por funcionar como parqueos para las y los funcionarios de las instituciones, con una capacidad física para dos o hasta más vehículos; estos espacios poseen bancas y pollitos; en una de las instituciones incluso se encuentra un kiosco, este tipo de estructuras durante las mañanas son utilizados para que los y las residentes pasen parte de su tiempo (Fuente: observaciones no participante, realizadas en el 2008, material original disponible).

En lo que respecta a los espacios bajo techo, la mayoría de los albergues tiene una 
sola construcción, en donde se encuentran oficinas, dormitorios, cocina, comedor, enfermería, área de terapia física, entre otras. La minoría de estos albergues poseen otras construcciones bajo techo, dentro de estas se encuentran pequeñas capillas, salas para terapia física, lavandería e incluso hasta la administración.

En estos espacios bajo techo, todos los albergues tienen lozas tipo cerámica en los pisos, con paredes de concreto, techos y cielorrasos presentes, algunos de ellos con espacios para recibir visitas, todos poseen un espacio para ver televisión. Asimismo, poseen un espacio, dentro de la planta principal o fuera de la misma, que funciona como el área de terapia física, la cual posee barras para deambular, sillas y mesas para diferentes actividades, entre otros recursos. Además, existen servicios sanitarios fuera de los dormitorios, grandes espacios abiertos que comunican dormitorios, comedor, enfermería y áreas administrativas. Las paredes presentan colores con tonos claros de celeste, verde agua, crema, beige, entre otros.

Todos los espacios en donde se encuentran los $y$ las personas adultas mayores poseen grandes ventanales, puertas muy amplias que pasan abiertas, ventiladores de techo $y$ pared en los lugares calientes; los espacios en donde hay dificultad para que ingrese la luz natural son iluminados con fluorescentes o bombillos.

En lo que respecta a la accesibilidad, cinco de los albergues poseen áreas con pendientes, las mismas con diferentes grados de inclinación; en estos espacios, así como, en algunos otros, por ejemplo, servicios sanitarios $y$ accesos a los parqueos, existen porciones de suelo que tienen una superficie antideslizante, la cual en la mayoría de los casos, se encuentra deteriorada. En todas las instituciones estos desniveles se acompañan de barandas para sujetarse por lo menos a un lado de la pendiente.

Las áreas verdes, en donde se encuentran los jardines, los kioscos, las bancas, o cualquier otra estructura de esta índole en todos los albergues, poseen un acceso directo a través de un camino, en algunos casos de cemento, otros de lozas, madera, entre otros, sin embargo, para ingresar a los espacios enzacatados existen barreras principalmente arquitectónicas, entre estas se encontraron rocas que funcionan como adornos, grandes flores, pequeñas rejas decorativas con formas de arcos, letreros con indicaciones como "Por favor deposite la basura en el basurero".

Las áreas o módulos que se encuentran separados de la planta principal de los albergues están cerradas bajo llave, igual situación se presenta cuando estos espacios se encuentran dentro de la planta principal; una funcionaria indica que la sala de terapia se abre solo cuando viene la terapeuta (Fuente: entrevista semi estructurada para funcionarios, 2008, material original disponible), además, un residente comenta que la iglesia solo se abre los jueves $y$ los viernes, cuando viene el padre (Fuente: entrevista semi estructurada para residentes, 2008, material original disponible).

En todos los albergues, la funcionalidad de los espacios no siempre concuerda con la finalidad de los mismos, debido a que hay espacios que cumplen diferentes funciones; por ejemplo, una funcionaria comenta que la parte de adelante es un patio, pero se utiliza de parqueo, para secar la ropa más difícil y como lugar para descargar la mercadería cuando llegan los almacenes (Fuente: entrevista semi estructurada para funcionarios, 2008, material original disponible). En un albergue se observó que el comedor es utilizado como sala de televisión, como centro de reunión para los y las residentes y sus visitas, entre otras.

En la mayoría de las instituciones, existen horarios establecidos para la utilización de algunos espacios para la recreación. Una funcionaria comentó que la sala de terapia se encuentra abierta los martes, jueves y sábados, cuando se encuentra laborando la terapeuta física (Fuente: entrevista semi estructurada para funcionarios, 2008, material original disponible). Sin embargo, existen espacios que no poseen un horario establecido. Un residente comentó que pueden estar en la sala cuando quieran, excepto cuando el personal se encuentra limpiando, por que es peligroso que se caigan (Fuente: entrevista semi estructurada para residentes, 2008 , material original disponible). 
Todos los albergues cuentan con un mínimo de un televisor y un radio destinados para el entretenimiento de los y las residentes. Además, la mayoría de los albergues poseen juegos de mesa como bingo o lotería, dominó, cartas o naipes y servicio de suscripción a algún periódico nacional. Todos los centros cuentan con mobiliario que se puede utilizar para la recreación: mesas, sillas, sillones, entre otros.

La mayoría de albergues poseen equipos $y$ materiales para la recreación que se utilizan en otras áreas; una funcionaria comenta que la terapeuta física tiene grabadora, lápices de color, crayolas, periódicos, tijeras, bolas y otros juegos que utiliza en sus sesiones de trabajo (Fuente: entrevista semi estructurada para funcionarios, 2008, material original disponible).

Con respecto a la regulación de la utilización de los equipos y materiales para la recreación, se encontró que en la mayoría de las residencias es una de las funciones que desarrolla el personal de terapia física, una funcionaria comenta que la asociación compra estos materiales y que es la profesional de terapia la que se encarga de ver cómo se utilizan (Fuente: entrevista semi estructurada para funcionarios, 2008, material original disponible).

Otros equipos que se mantienen con libre acceso, como son los televisores y los radios, en la mayoría de los albergues, son regulados por la administración de cada centro; un residente menciona que solo se puede ver televisión después del almuerzo y que los domingos en la mañana se pone la misa (Fuente: entrevista semi estructurada para residentes, 2008, material original disponible); una funcionaria expone que por indicación de la administradora, el televisor hay que apagarlo a las 6:00 pm y solo los miércoles o sábados en la noche cuando hay partidos de fútbol hay permiso de mantenerlo encendido (Fuente: entrevista semi estructurada para funcionarios, 2008, material original disponible).

De acuerdo con la observación realizada, en todos los albergues existen diferentes espacios para guardar los equipos y materiales para la recreación; sin embargo, en todos existe en el área destinada para que trabaje la o el profesional de terapia física espacios para almacenarlos, entre estos se encuentran armarios, cajones, escritorios.

En los centros existen televisores, radios, sillas, mesas, entre otros que no se guardan, sino que por el contrario, se encuentran a simple vista. Sin embargo, estas instituciones poseen bodegas destinadas para el almacenamiento de materiales o equipos de diferente índole, en las cuales se mantienen algunos equipos o materiales para la recreación, ante esto, una funcionaria comenta que en la bodega de lata tiene guardado un juego de bolos, naipes, títeres y una guitarra, entre otras cosas, debido a que como nunca se utilizan es mejor mantenerlas bajo llave para que no se deterioren (Fuente: entrevista semi estructurada para funcionarios, 2008, material original disponible).

Con respecto a la frecuencia de la utilización de los equipos y materiales para la recreación, se encontró que en la mayoría de los albergues el televisor, el radio y los periódicos se usan todos los días, incluyendo fines de semana. Los juegos de mesa y manualidades son utilizados muy poco, principalmente cuando se encuentra el o la profesional en terapia física, o bien, cuando llegan grupos organizados como universidades. Las crayolas, las tijeras, los papeles, la plasticina, las bolas, entre otros, se utilizan frecuentemente, en especial, cuando participan en la sesión de terapia física.

\section{DISCUSIÓN}

A continuación se describe la confrontación de los resultados obtenidos con la teoría existente acerca de cada uno de los datos analizados; la información se estructuró según las categorías del estudio.

\section{A.1. MISIÓN, VISIÓN Y PROPÓSITOS DE FUNCIONAMIENTO}

De acuerdo con lo verbalizado por las funcionarias y por los y las residentes, existe un desconocimiento de cuál es la misión y la visión del funcionamiento de los albergues. Sin embargo, los sujetos sí conocen el propósito por el que se establecieron estas instituciones, haciendo referencia específicamente al propósito de satisfacer las necesidades básicas; con 
base en lo indicado por los sujetos, la recreación no es considerada una necesidad básica a ser satisfecha por las instituciones. Esto difiere con lo establecido por el CONAPAM, ya que Valverde (2004) menciona que esta institución plantea que los servicios básicos que deben brindar los albergues a los y las residentes son: alimentación, lavandería, habitación, actividades recreativas, ocupacionales y culturales, actividades físicas, estimulación mental y contacto social.

El hecho de no incluir la percepción de la recreación como una necesidad básica puede ser consecuencia de diferentes factores; primero, la influencia del bajo nivel académico de los sujetos. Esta variabilidad en el acceso a la educación formal es fundamental desde un punto de vista fenomenológico, debido a que la cercanía en las relaciones interpersonales que se establecen entre el personal y la población adulta mayor, posee una dinámica influenciada por la posibilidad de entender los comportamientos de los y las residentes por parte de las funcionarias, por ende, al existir un conocimiento previo de una conducta, esta puede ser acompañada de una manera más adecuada; al respecto Nuñez (2002) señala que durante la convivencia de personas de diferentes grupos etarios, se suele recurrir a experiencias o vivencias formales 0 informales para aclarar situaciones ajenas 0 incomprensibles a la dinámica que se presenta. Por ende, considerando únicamente el acceso a la educación se puede esperar que el acompañamiento hacia los y las residentes difiera de una persona a otra, y la finalidad o razón del por qué se da este acompañamiento, también sea diferente.

Como se mencionó anteriormente para todos los albergues, el personal ha recibido alguna capacitación no formal en temas relacionados con el acompañamiento de la persona adulta mayor, lo que permite establecer según Nuñez (2002), que la población de funcionarias puede realizar sus labores de una manera más cercana a las y los residentes, ya que su interacción se ve facilitada por el conocimiento previo de la mayoría de las situaciones que experimentan estos y estas últimas, durante la vida diaria; sin embargo, estas capacitaciones en la mayoría de los casos no profundizan en temas relaciona- dos con el uso creativo del tiempo libre o bien con la recreación.

En segundo lugar, las funciones del personal se encuentran orientadas a brindar la satisfacción de las necesidades básicas de aseo e higiene, vestimenta, alimentación y control médico; sin embargo, no existe ningún miembro del personal que se encargue de la realización de actividades durante el tiempo libre de los y las residentes, por lo tanto, el uso creativo del tiempo libre no es percibido como una necesidad básica, lo que implica que existe un desconocimiento o falta de información por parte del personal de los albergues con respecto a la importancia de la recreación para el mejoramiento de la calidad de vida de las personas adultos mayores.

\section{A.2. CANTIDAD DE TIEMPO LIBRE}

Según los resultados obtenidos, en promedio una persona adulta mayor residente de albergue posee entre cinco a siete horas de tiempo libre, durante el cual no realizan actividades de sueño $y$ descanso, ni asisten a misa ni reciben alimentos, entre otras, lo cual coincide con Lopategui (2001), en su definición de tiempo libre, como la finalización de actividades obligatorias; lo cual representa el tiempo que queda libre una vez terminado el trabajo y cubiertas las necesidades casi "biológicas". Tiempo que sobra luego de las obligaciones formales del trabajo y necesidades de la vida. Es aquella porción del tiempo de un individuo que no está dedicada al trabajo o a las responsabilidades relacionadas con el trabajo $u$ otras actividades necesarias para subsistir y que por lo tanto, se pueden considerar como tiempo libre $y$ se pueden utilizar arbitrariamente. El tiempo libre puede conseguirse si se suman las horas dedicadas a las necesidades básicas y luego se le restan a las veinte y cuatro horas que constituye un día.

Los resultados obtenidos concuerdan con los hallazgos en el estudio de Page (1996), quien comenta que las personas adultas mayores de los 64 años poseen entre cuatro y ocho horas de tiempo libre. Page (1996) menciona que esta cantidad de tiempo se incrementa de manera directamente proporcional con la edad, 
debido a que a mayor edad se dedica menos tiempo para satisfacer personalmente sus necesidades individuales.

Desde la vivencia de ambos grupos de sujetos, existe una forma variada de percibir cómo los y las residentes utilizan el tiempo en diferentes actividades durante el día; sin embargo, es consistente que cada estrato percibe la existencia del tiempo libre, con dos características fundamentales, en primer lugar, es extenso en su cantidad $y$ de poca utilidad en su finalidad, debido a que por sus historias de vida $y$ por los estilos de vida actuales se perciben poco productivos, Nuñez (2002) comenta que desde la fenomenología de la cotidianidad, en Costa Rica, el tiempo o su uso se percibe según lo que produzcamos en su utilización.

\section{A.3. ACTIVIDADES, PROGRAMAS Y PROYECTOS PARA EL USO CREATIVO DEL TIEMPO LIBRE}

Con respecto a los resultados obtenidos sobre el uso del tiempo libre por parte de las $y$ los adultos mayores, se desprende que los y las residentes pasan la mayor parte de su tiempo libre sentados (as) sin hacer ninguna actividad creativa o viendo televisión, lo cual difiere con las normas establecidas de acreditación de estos centros, ya que el Ministerio de Salud (2006), a través de estas normas establece, como uno de los deberes de la institución para la persona adulta mayor, el de mejorar la calidad de vida del residente $y$ usuario mediante la creación $y$ ejecución de programas de actividades recreativas en el centro diurno y el hogar.

Los datos relacionados con el uso creativo del tiempo libre, sugieren que existe una monotonía en las actividades que realizan las personas adultas mayores institucionalizadas, situación que concuerda con los hallazgos del estudio realizado por Bell et ál. (1999), en el que encontraron que las personas adultas mayores institucionalizadas dedican más del 94,4\% del tiempo libre a pasar sentados en sillones, butacas, sillas y mecedoras, en donde la actividad más común es la de mirar televisión.

Destacando el efecto de estas actividades, se consideran no creativas, por lo que no hay un uso creativo del tiempo libre, ya que Franch y Martinell (1994), plantean que las actividades creativas durante el tiempo libre le brindan a la persona espacios para la socialización, la educación, la prevención y se convierten en un espacio trasformador de la realidad.

Desde la vivencia diaria externada por el personal y los y las residentes, hay dos aspectos fundamentales que considerar; Hidalgo (2001) plantea que desde una visión sociológica las actividades cotidianas se convierten en estructuras, ya que lo que se repite reiteradamente en un orden social se establece como "normal", o establecido, al considerar que existe una reiteración en las actividades diarias en los albergues, así como, en la finalidad de las mismas; estas y sus actores conforman una estructura, la cual no incorpora actividades para el uso creativo del tiempo libre ya que no son vistas como fundamentales en el funcionamiento de los centros.

Page (1996) plantea que al experimentar los cambios sociales y las transiciones, existen gran cantidad de experiencias que se han adquirido desde la infancia hasta la vejez y se incorporan como parte de la vida diaria, si el uso creativo del tiempo libre no ha sido incorporado en este balaje durante la historia de vida de cada residente $y$ de cada funcionaria, es posible que este no se incorpore dentro de la estructura social y cotidiana actual de los albergues.

Desde la fenomenología de la cotidianeidad, Núñez (2002) establece que en los cuidados a largo plazo de las personas adultas mayores en Costa Rica, intervienen la percepción de la persona adulta mayor como tal $y$ la de las personas que la acompaña en sus diferentes procesos, en donde la percepción de cada uno de los actores hacia el otro es fundamental.

Bolaños (2005), en su tesis concluye que en estas instituciones las personas presentan una desvalorización social de este grupo poblacional, así como, una deformación en su rol social y se les encuadra en una posición de que el periodo de la vejez es para descansar, dormir y esperar, por lo que el tiempo libre se orienta en que los y las residentes pasen sentados en sillones, mecedoras, acostados en sillones, sentados $y$ sentadas en sus sillas de ruedas en filas, entre otras; situación que imposibilita que 
ese tiempo libre se utilice en actividades en la que la persona adulta mayor pueda experimentar momentos creativos que le permitan desenvolverse en un ambiente más inclusivo y cooperativo para lograr mejorar su estado físico, psicológico y social.

La teoría social del desligamiento según Burke y Walsh (1998), plantea que la persona al envejecer modifica el equilibrio en sus relaciones con la sociedad, ya que por él o ella misma, o bien, por la sociedad, la persona adulta mayor rompe o altera la calidad en las relaciones sociales y por ende, en los comportamientos colectivos, e incluso se asumen roles o papeles para mantener este equilibrio, situación que es evidente con las rutinas y la exclusión de las actividades recreativas durante el día; por el contrario, la persona adulta mayor asume una postura de espera, ya que usualmente está a la expectativa de que el personal de las instituciones realice las tareas por ellos y ellas, por ejemplo, trasladarlos al comedor, bañarlos, prepararles los alimentos, entre otras.

\section{A.3.1. Actividades recreativas}

Por otro lado, los sujetos coincidieron en el aspecto de frecuencia en la realización de actividades recreativas, que en la mayoría de las veces, estas son realizadas por grupos organizados ajenos a la institución y son por motivo de celebraciones o épocas festivas.

De lo anterior, se destaca que la recreación en los albergues no se está dando de forma regular, lo que difiere un poco con la Ley 7935 , planteada por la Asamblea Legislativa (1999), que establece que toda persona adulta mayor tendrá derecho a una mejor calidad de vida, mediante la creación y ejecución de programas que promuevan la participación en actividades recreativas, culturales $y$ deportivas promovidas por las organizaciones, las asociaciones, las municipalidades y el Estado.

Este incumplimiento de actividades recreativas podría deberse a la falta de información por parte de los albergues sobre estos programas, o por la falta de divulgación y control del cumplimiento de las leyes de las institu- ciones encargadas de velar por los mismos, por lo que se evidencia una falta de sistematización sobre cómo se están realizando las actividades en los albergues. También, la ubicación de los albergues es un factor a considerar, ya que en la mayoría de los casos, los lugares en donde estos centros se encuentran ubicados es de muy difícil acceso, o bien, en lugares de reconocida peligrosidad comunal, por ejemplo, en barrios urbano marginales, en sitios alejados de los caminos principales y del servicio de transporte público, en cuestas o en lugares poco visibles, lo que puede limitar que las y los residentes salgan de los centros, que personas o grupos se trasladen o incluso, se enteren de la existencia de estas instituciones.

\section{A.4. PERSONAL ENCARGADO DE LA REALIZACIÓN DE ACTIVIDADES RECREATIVAS}

De acuerdo con la opinión de las funcionarias y los residentes de los albergues, con respecto a la persona encargada de realizar actividades recreativas, coinciden en que no existe una persona responsable de realizar dichas actividades, además de la información obtenida, se desprende que los albergues solo cuentan con el encargado(a) de terapia física y no existe ninguna persona que se encargue de desarrollar actividades de recreación en estos centros de forma regular.

Lo anterior es preocupante, ya que el Ministerio de Salud (2006) plantea como misión de los albergues, garantizar que la calidad de los servicios que se brindan, se realice en forma eficiente $y$ eficaz, mediante la participación del equipo interdisciplinario, para contribuir a mejorar la calidad de vida de la persona adulta mayor. Esto último es apoyado por Valverde (2004), quien se refiere al derecho de mejorar la calidad de vida del residente y usuario mediante la creación y ejecución de programas de actividades que pueda brindar el centro.

De acuerdo con el CONARE (2008), a nivel nacional existe una amplia oferta académica relacionada con formación en recreación, movimiento humano y uso creativo del tiempo libre, 
la que permite al país contar con profesionales formados tanto a nivel privado como público, en las competencias antes mencionadas y por ende, existe una oferta profesional necesaria para satisfacer la demanda laboral de los albergues para personas adultas mayores.

\section{A.5. RECURSOS MATERIALES Y CAPACIDAD ESTRUCTURAL Y FÍSICA PARA LA RECREACIÓN}

De acuerdo con la información recolectada sobre los espacios físicos para la recreación, se encontró que estos son subutilizados o mal utilizados. En cuanto a las normas que un albergue debe cumplir, con respecto a los espacios para el ejercicio físico, el Ministerio de Salud (2001) plantea que el establecimiento debe contar con acceso a un espacio o salón multiuso, donde se puedan llevar a cabo actividades de terapia física de los usuarios(as) (actividad física, ejercicio físico). Al no existir dicho acceso a lugares para recrearse, se le limita la posibilidad a las personas adultas mayores de poder realizar actividades para su bienestar biopsicosocial en su tiempo libre, por lo que se hace crítico el habilitar este tipo de espacios para maximizar los trabajos a realizar con los participantes adultos mayores y así, incrementarles su calidad de vida.

Con respecto a los materiales utilizados para la recreación, con base en la observación no participante, se encontró que la mayoría de los albergues cuenta con dichos instrumentos; sin embargo, estos normalmente se encuentran guardados bajo llave o en la sala de terapia y esto dificulta que los adultos mayores tengan la posibilidad de utilizarlos en su tiempo libre. Estos materiales, según el Ministerios de Salud (2006), en sus normas de acreditación a los albergues, deben estar accesibles para que los adultos mayores puedan tener tiempo de esparcimiento y relaciones sociales al utilizar estos con otros adultos mayores. Es de gran importancia para esta población tener acceso a materiales de juego y de ejercicio, entre otros para mejorar su estado físico, psicológico y social y por ende, mejorar su calidad de vida.
Hay dos aspectos importantes a considerar desde la vivencia externada por los sujetos, en primer lugar, los recursos y materiales se encuentran destinados a la satisfacción de las necesidades básicas, por ende, al no percibir la recreación como una de estas no se visualizan estos recursos con esa finalidad fundamental, Nuñez (2002) comenta que lo que se utiliza en la cotidianeidad se encuentra ligado a lo que se desea producir en la misma, y por ende, si no se utiliza creativamente el tiempo libre, o no se realizan actividades recreativas con dicho fin, no se necesita utilizar materiales o recursos y por ende, no son necesarios en las actividades cotidianas.

En segundo lugar, y con respecto a la accesibilidad, Nuñez (2002) plantea que existe desde la fenomenología de la cotidianidad una disputa entre lo privado y lo público, que limita el acercamiento a estos espacios o recursos. Al encontrar cierta limitación de acceso o acercamiento a los espacios o recursos para la recreación, las personas adultas mayores e incluso el personal pueden percibir que estos no son adecuados, permitidos o incluso propiedad de los y las residentes y por lo tanto, su uso restringido es adecuado.

\section{CONCLUSIONES}

Con base en los resultados obtenidos del estudio y el análisis de los mismos, se exponen a continuación las principales conjeturas alcanzadas durante el desarrollo de la investigación.

a) En la conceptualización de las necesidades básicas para satisfacer a las personas adultas mayores que residen en albergues, existe la exclusión de la recreación como parte de estas necesidades.

b) La distribución del tiempo durante las diferentes actividades del día, permite que una persona adulta mayor residente de estas instituciones, cuente con un tiempo adecuado para la participación en todas las actividades orientadas a la satisfacción de sus necesidades básicas; sin embargo, a pesar de la disponibilidad 
de tiempo libre, este no se utiliza en la mayoría de los casos, en actividades para la recreación y el uso creativo del tiempo libre.

c) Durante los espacios de tiempo libre que disponen los y las residentes de las instituciones, no se realiza un uso creativo de su tiempo, por el contrario, las actividades que implementan no son potencializadoras de un envejecimiento integral.

d) La frecuencia en la realización de actividades recreativas se encuentra condicionada en la mayoría de los casos a espacios de tiempo libre del personal, o bien, a la presencia de agentes externos como grupos académicos, religiosos, voluntarios, entre otros; sin embargo, estas actividades poseen una estrecha relación con la celebración de algunas festividades, como el Día del Padre, Día de la Madre, entre otras.

e) En la distribución de funciones del personal, existe la ausencia en la delimitación de la responsabilidad de la realización de actividades recreativas. El personal se caracteriza, en términos laborales, de poseer una enorme capacidad de polifuncionalidad, debido a la variedad de tareas que cumplen en los albergues; sin embargo, dentro de estas se encuentra ausente las relacionadas con la recreación por lo que al no haber una persona encargada específicamente para esta $y$ al no ser parte de las funciones del personal, se da por entendido que la recreación no se realiza en los albergues de una forma regular ni es prioridad.

f) Existe una subutilización de los recursos físicos y materiales encontrados en los albergues a nivel nacional, incluso los fines actuales de uso se convierten en limitantes para que las y los adultos mayores utilicen estos espacios para implementar actividades durante su tiempo libre.

g) En cuanto al uso creativo del tiempo libre y la recreación, existe un divorcio entre las prácticas de funcionamiento utilizadas en los albergues del país y las políticas y normativas vigentes que favorecen la satisfacción de las necesidades básicas de las y los residentes.

\section{RECOMENDACIONES}

Con base en las conclusiones obtenidas del estudio y el análisis de las mismas, se exponen a continuación y a modo de cierre, las principales recomendaciones relacionadas con el proceso de investigación realizado.

a) Dentro de las condiciones socio demográficas que caracterizan a los y las funcionarias de los albergues, se debe considerar el nivel académico $y$ de formación, ya que un componente educativo $y$ de sensibilización hacia el personal permite una mejor actuación de los diferentes actores del modelo.

b) Dentro de las actividades realizadas por los y las residentes durante el día, se les debe orientar acerca de la definición del uso creativo del tiempo libre, además de brindar espacios para que las personas expresen sus dudas e ideas acerca de formas diferentes para utilizar su tiempo libre.

c) Las instituciones y las comunidades en general, deben brindar a las personas residentes, opciones variadas para la utilización creativa del tiempo libre, que considere el potencial estructural y material de los centros.

d) Se debe asignar dentro de las funciones del personal, un rubro de actividades relacionadas con el uso creativo del tiempo libre.

e) Deben fomentarse espacios y toda clase de facilidades que permitan a la persona adulta mayor realizar ejercicio físico, a través del cual pueda contribuir en el mejoramiento de su calidad de vida.

f) La práctica regular de actividades recreativas, debe incorporarse en los espacios para la utilización creativa del tiempo libre, brindando desde la comunidad y la institución, la mayor cantidad de facilidades para la práctica y la adherencia a esta conducta. 


\section{BIBLIOGRAFÍA}

Asamblea Legislativa. "Ley Integral para la persona adulta mayor 7935”. Alcance 88 La Gaceta 221. San José, Costa Rica. 15 de noviembre de 1999.

Bolaños, C. "La calidad universitaria desde una perspectiva de género". Actualidades Investigativas en Educación 5 . Universidad de Costa Rica, 2005. [Revista digital] En: <http://revista.inie.ucr.ac.cr/ articulos/extra-cea/archivos/calidad.pdf> [Consultado el $1^{\circ}$ de marzo de 2010].

Brigeiro, M. “'Envejecimiento exitoso' y 'tercera edad': problemas y retos para la promoción de la salud". Invest. Educ. Enferm. 23 (1). 2005: 102-109.

Buendía, J. Envejecimiento y Psicología de la Salud. Madrid, España: Siglo XXI de España, 1997.

Burke, M. y Walsh, M. Enfermería Gerontológica. Cuidados integrales del adulto mayor. 2 ed. España: Harcourt Brace El Sevier SA, 1998.

Consejo Nacional de Rectores. Cifras relevantes de las universidades estatales, posibilidades de estudio en la educación superior estatal de Costa Rica en el 2008. 2008. [En línea]. En: <http://www. conare.ac.cr/index.php?option $=\mathrm{com}_{-}$ r e m o s it ory \& I t e m i d = $134 \&$ func $=$ startdown $\& \mathrm{id}=2>$ [Consulado el $1^{\circ}$ de marzo de 2010].

Fernández, R. "Vejez con éxito o vejez competente. Un reto para todos". [Ponencia realizada en el Simposio Internacional Envejecimiento Competente, Retiro y Seguridad Social]. Bogotá, Colombia. 1999.

Hidalgo, J. El envejecimiento: aspectos sociales. San José, Costa Rica: Universidad de Costa Rica, 2001.

Lopategui, E. Recreación. 2001. [En línea]. En: $<$ www.saludmed.com/Bienestar/Cap6/ Recrear.html> [Consultado el 28 de julio de 2008].

Millán, J. Principios de Geriatría y Gerontología. Madrid, España: Mc Graw Hill Interamericana, 2006.
Ministerio de Salud. "Manual de Normas para el funcionamiento de establecimientos de atención integral a las personas adultas mayores". La Gaceta 52. San José, Costa Rica. 09 de agosto de 2001.

Ministerio de Salud. Estado de salud de la persona adulta mayor. San José, Costa Rica. 2004.

Ministerio de Salud. Normas para la acreditación de establecimientos de atención integral a la persona adulta mayor (Centro Diurno-Hogar). San José, Costa Rica. 2006.

Ministerio de Salud. Dirección de Servicios de Salud. Unidad de Acreditación. Manual de Normas para la acreditación de establecimientos de atención integral a la persona adulta mayor. 1ed. República de Costa Rica, 2006.

Nuñez, D. Vejentudes y juventudes. La familia en esta (h)orilla del siglo XXI. San José, Costa Rica: EUNED, 2002.

Organización Panamericana de la Salud. "La salud de las personas adultas mayores en Costa Rica”. Serie Análisis de Situación de Salud 56 (16) nro. 12. San José, Costa Rica: Organización Panamericana de la Salud, Ministerio de Salud, Consejo Nacional de la Persona Adulta Mayor, 2004

Proyecto Estado de la Nación (Costa Rica). Resumen Decimotercer Informe Estado de la Nación en Desarrollo Humano Sostenible. San José, Costa Rica: Programa Estado de la Nación, 2007.

Rico, C. "Recreación y adulto mayor. La tercera edad en el Plan Nacional de Recreación". [Ponencia presentada en el 1er. Congreso Nacional de atención integral al adulto mayor]. Ministerio de Salud de Colombia. Bogotá. 1999. [En línea]. En: $<$ http://www.redcreacion.org/articulos/ terceraedad.html> [Consultado el 17 de julio de 2008].

Rodríguez, R. et ál. Geriatría. México: Mc Graw Hill Interamericana Editores SA, 2000.

Valverde, J. "Contexto y perfil de las Instituciones de Bienestar Social para 
la atención de las personas adultas mayores". Documento elaborado para el CONAPAM. 2004. [En línea] En: $<w w w . c o n a p a m . g o . c r / c o n t e x t o . p d f>$ [Consultado el 30 de julio de 2009].
Wilmore, J. y Costill, D. Fisiología del esfuerzo $y$ del deporte. $5^{\text {a }}$ ed. Barcelona, España: Paidotribo, 2004.

Fecha de ingreso: 24/08/2010

Fecha de aprobación: 24/01/2011 